\title{
Arguing over Actions that Involves Multiple Criteria: A Critical Review
}

\author{
W. Ouerdane, N. Maudet and A. Tsoukias \\ LAMSADE, Univ. Paris-IX, Place du Marchal De Lattre de Tassigny, 75775 Paris \\ Cedex 16 \\ \{wassila.ouerdane, maudet, tsoukias\} @lamsade.dauphine.fr
}

\begin{abstract}
There has recently been many proposals to adopt an argumentative approach to decision-making. As we feel that the underlying assumptions made in these different approaches is not always clearly stated, we reviewed these works, taking a more classical decision theory perspective, and even more precisely a multicriteria perspective. It appears that these approaches seem to have much to offer to decision models, because they allow more expressivity in the specification of agents' preferences, because they naturally cater for partial specification of preferences, and because they make explicit many aspect that are usually somewhat hidden in decision models. On the other hand, the typically intrinsic evaluation used in these approaches is not always the most appropriate, and it is not always clear how the multicriteria feature is taken into account when it comes to aggregating several arguments that may potentially interact and refer to different criteria. ${ }^{1}$
\end{abstract}

\section{Introduction}

Decision-support systems aims at helping the user to shape a problem situation, formulate a problem and possibly try to establish a viable solution to it. Under such a perspective decision support can be seen as the construction of the reasons for which an action is considered a "solution to a problem" rather than the solution itself (see [Tso]). Indeed the problem of accountability of decisions is almost as important as the decision itself. Decision support can therefore be seen as an activity aiming to construct arguments through which a decision maker will convince first herself and then other actors involved in a problem situation

\footnotetext{
${ }^{1}$ A previous version of this work has been presented at the LCD07 workshop.
} 
that "that action" is the best one (we are not going to discuss the rationality hypotheses about "best" here). Decision Theory and Multiple Criteria Decision Analysis have focussed on these issues for a long time, but more on how this "best solution" should be established and less on how a decision maker should be convinced about that (for exceptions on that see $\left[\mathrm{BMP}^{+} 00\right]$, [BS02]).

More recently, in the field of artificial intelligence, argumentation has been put forward as a very general approach allowing to support different kinds of decision-making [BG96, PV02, Pol87, PJ98, AP06]. Typically, one will construct for each possible decision (alternative) a set of positive arguments (e.g. good consequences of the decision), and a set of negative arguments (e.g. bad consequences of the decision). However, decision-makers do not simply list pro and cons: they exchange arguments, some of them interacting with others, attacking or reinstalling previous arguments put forward (by the other party, or sometimes indeed by itself). Distinguishing what eventually should count as acceptable arguments has been the study of numerous studies, and necessitates to value the arguments. Cayrol and Lagasquie-Schiex [CLS05] distinguish intrinsic valuation of arguments (without any consideration for the other arguments -for instance it may be based on the credibility of the source), and interaction-based valuation of arguments (simply resulting of the interactions between arguments -for instance some may be better supported than others, etc.). In the seminal work of Dung [Dun95], different semantics are proposed, which interpret differently what (sets of, in this case) arguments should be considered acceptable, only based on their interaction-based valuation. More recently, some approaches propose to take both aspects into account, see e.g. [KP98]. Once the valuation has been made, it is then possible to select the acceptable arguments. Usually, only a crisp selection is allowed: arguments are acceptable or not; however a more gradual acceptability is also possible [CLS05]. Our objective in this paper is to clarify the connections between argumentation and decision-making, and more precisely to inspect the recent proposals that have been put forward to handle (multi-criteria) decision-making in an argumentative framework.

The rest of this paper is as follows. In Section 2, we examine more carefully what it means to argue for an action, and in particular we try to see how the litterature account for the fact that actions may take different scores over different criteria. We confront the different proposals put forward in the litterature to our multicriteria perspective, discuss some hidden assumptions that they make, and propose a new argument scheme. In Section 3, we move on to the following step by inspecting how aggregation should be performed.

\section{Arguing over Actions}

We start by identifying (i) what makes decision over actions different to decision over beliefs, (ii)what means an argument is in favour of an action, and (iii)how the literature account for the fact that action may take different scores over different criteria. 


\subsection{The specificity of argumentation over action}

Argumentation is usually conceived as a process for handling (potentially conflicting) beliefs. In AI, many systems have been proposed that allow to capture the non-monotonic nature of this kind of reasoning. As a result, the basic building block, the argument, can typically be defined as a premise/conclusion pair, whereby you state that that conclusion should be reached under these premises. What is discussed here is the truth-value of the conclusion, so an argument supporting a conclusion basically asserts some evidence to believe that this conclusion holds.

When it comes to decision-making though, this rather crude argument scheme that we define here needs to be refined. Indeed, as it has been recognized for a long-time now, there is a significant difference between argumentation for beliefs and argumentation for actions [FP97, FP98].

\subsection{How arguments support actions?}

Now we come to what is, perhaps, the central question here: what does it mean exactly that "an argument is in favour of an action $a$ "? The intuitive reading is that action $a$ will have "good consequences". So you must first somehow valuate the outcome of the action. In decision models, this will be typically done by using an ordered scale defining the different values that can be used to assess the action (for instance, marks from 0 to 20 for students). Now what counts as a positive or negative outcome is specific to each agent, and depends of its (subjective) preferences. That is, you must classify the outcome of the actions. To do that, you must use a bipolar scale. In decision models, one classical approach is that the agent must then use the evaluation scale and specify a frontier, that is, a neutral point (or zone), thus inducing a bipolar scale. This will in turn allow us to determine what counts as an argument pro or an argument against the action. For instance, I would say that according to "marks", we have an argument in favour of the student if its more than 12 .

\subsection{The need for different "points of view"}

Intuitively, as we said before, performing an action will bring about a state of the world which will be judged desirable or not. In general however, it is possible that you may have different valuations that you assign to a given action, depending on different points of view that you take to evaluate that action. Very often, these different valuations cannot be merged into a single point of view. This has been recognized in particular in multi-criteria decision-making, where a criterion is regarded as a point of view against which it is possible to compare different actions. Now, the definition of the neutral point for each point of view defines what is called a profile. So the profile plays the role of a special action against which each action will be compared. 


\subsection{Discussion of existing approaches}

In [FP97], Fox and Parsons proposed one of the first account that tried to advocate an argumentative approach to decision-making. They recognize and clearly state what makes argumentation for actions different from argumentation for beliefs. They put forward the following argument scheme:

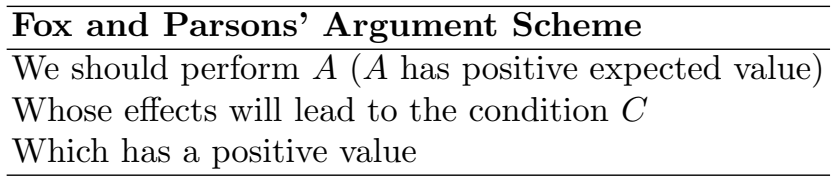

As explained by Fox and Parsons, the advantage of this representation is that it makes explicit three inference steps: (i) that $C$ will indeed result from action $A$, (ii) that $C$ has some positive value, and (iii) that $A$ has a positive expected value. Clearly, steps (ii) and (iii) requires additional information in order to be able to assign values to situations, and to decide whether the action has indeed a positive expected value. The valuation of the condition is subjective (dependent of the agent's preference), and represented here by "labelling the proposition describing $C$ with a sign drawn from a dictionary", which can be qualitative or not and plays the role of a scale. Interestingly, they also allow for different points of view over which values can be assigned. So for instance, opting for a given candidate could lead to the consequence the chosen candidate has a mark over 12 , fact that we consider positive. This would be represented as the following assignment

$$
\text { mark } \geq 12: v a:+
$$

meaning that the condition mark $\geq 12$ is positively evaluated by our agent (it count as a positive argument), where $v a$ is the justification for this value assignment. Although this aspect is not deeply explored in the paper, a very interesting feature of this approach is then that it makes explicit the grounds allowing to assign this value to this condition: so what may count has obvious candidate to justify this value assignment, if we take the view of the multicriteria-decision approach, would be the user's preference profile ("I consider that the mark is good from 12"), as well as the preference model used ("I consider this to be a positive argument as long as it is beyond the limit previously stated"). On the other hand, this approach does not really acknowledge that actions themselves can be evaluated against a number of meaningful, predefined, dimensions: in fact, each condition induces a new dimension against which the action can be evaluated.

One of the most convincing proposal recently put forward to account for argument-based decision-making is the one by Atkinson et al. [ABCM06, Atk06]. They propose an extension of the "sufficient condition" argument scheme proposed by Walton [Wal96]. 


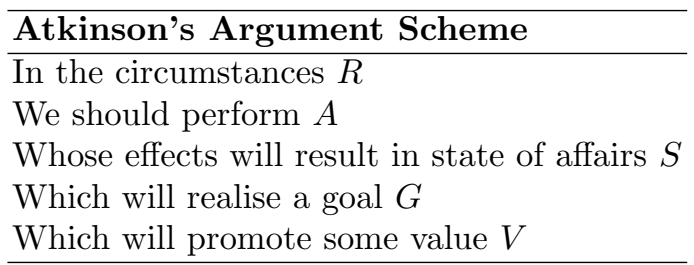

To avoid confusion with the previous approach, we must first make clear that the notion of value used here is quite different. As we shall see, it plays a role comparable to that of a criteria in multi-criteria decision making. Atkinson explains [Atk05] that values should not be confused with goals as "they provide the actual reasons for which an agent wishes to achieve a goal". Goals refer to single values, but an action can bring about a state of affairs that satisfy many goals, hence affecting different values.

Technically, a function value maps goals to pairs $\langle v$, sign $\rangle$ where $v \in V$, and sign belongs to the scale $\{+,-,=\}$ (but Modgil [Mod06] adds a notion of degree to which the value is promoted). For instance, using our running example, we could have

$$
\operatorname{value}(\operatorname{mark} \geq 12)=\langle\text { academic_level },+\rangle
$$

meaning that the value (criteria) academic quality is promoted when the mark is over 12 .

In this approach, values clearly play the role of criteria. So it looks like specifying goals amounts to specifying a (potentially partial) profile. However, the declarative nature of goals allows for more subtle classifications than what we typically have in decision models ${ }^{2}$. For instance, it is possible to express that

$$
\text { value }(\text { age } \geq 18 \wedge \text { age } \leq 32)=\langle\text { youth },+\rangle
$$

the value "youth" is only promoted when the age falls between 18 and 32 . It is also important to note that values are eventually assigned to state of affairs via goals. So the justification of value assignment to states is implicitly given by the fact that the goal is reached (or not). One potential problem is that it does not leave any other option if we were to provide some additional justification (for instance related to preference model used).

In [ABP05], Amgoud et al. propose an approach explicitly linking argumentation to multi-criteria decision-making. They see an argument as a 4 -tuple $\langle S, x, c, g\rangle$ where

$-S$ is the support of the argument,

$-x$ is the conclusion of the argument (the action)

$-c$ is the criterion which is evaluated for $x$,

$-g$ is the goal and represents the way $c$ is satisfied by $x$

\footnotetext{
${ }^{2}$ Although some approaches try to overcome these limitations, see for instance [aC96]
} 
It is required that $S$ is consistent when we add the fact that the action $x$ has taken place. Here, in a way that is reminiscent of the previous approach, each goal $g$ is explicitly associated to a criterion by means of a propositional formula $g \rightarrow c$, although the possibility of having goals referring to different criteria is also mentioned. In this approach, unlike in [Atk05], the use of (bipolar) scale is explicitly mentioned: the goals will fall either on the negative or on the positive side. Their approach also allows for quantitative measure of how good are the attained goals. So for instance, we may specify that knowledge base has several strata

$$
\begin{aligned}
& G_{2}^{+}=\{\text {mark } \geq 16\} \\
& G_{1}^{+}=\{16>\text { mark } \geq 12\} \\
& G_{1}^{-}=\{\text {mark }<12\}
\end{aligned}
$$

which means that the marks are considered as "good" from 12, and even "very good" from 16, while it is unsufficient when it is below 12. This comes together with formulae of the form

$$
\text { mark } \geq 16 \rightarrow \text { academic_level }
$$

which explicitly states that the goal $G_{2}^{+}$affects the criteria "academic level". Now each decision will have some consequences, that will in turn fulfill some goals or not. It is then possible to identify arguments pro and cons a given decision $x$, by simply scanning the knowledge base and checking which positive (resp. negative) goals are satisfied by the occurrence of that decision $x$.

In a very recent proposal, Morge and Mancarella in [MM07] propose a multiattribute argumentation framework for opinion explanation. Here, a main goal is split into sub-goals and so on. They make a distinction between high level goals ("abstract goals that reveal the user's need"), and low-level goals ("criteria for evaluating different alternatives").

As for the satisfaction of a goal by a given decision, this is explicitly stated by:

- decision rules of the form $R: g \leftarrow D, B_{1}, \ldots, B_{n}$ meaning that the goal $g$ can be achieved by decision $D$, given that conditions $B_{1}, \ldots, B_{n}$ are satisfied.

- goal rules of the form $R: g \leftarrow g_{1}, \ldots, g_{n}$ meaning that the head of the rule is reached if the goals listed in the body are reached

The notion of priority between rules allows to refine decision rules, in order to make more complex aggregation. So for instance; if we were to specify that we would chose an alternative if it meets one out of two goals, we would specify that

$$
\begin{aligned}
& R_{0}: g_{0} \leftarrow g_{1}, g_{2} \\
& R_{1}: g_{0} \leftarrow g_{1} \\
& R_{2}: g_{0} \leftarrow g_{2}
\end{aligned}
$$


with $R_{0} \succeq\left\{R_{1}, R_{2}\right\}$

Now if we inspect what plays the role of a criteria in this approach, it is difficult to say. In fact, there is no notion properly corresponding to that of a criteria: there is no point of view against it is possible to compare alternatives. It would be tempting to say that there exists an implicit preference model stating that the decision-maker prefers to satisfy goals, rather than not. However it is deceptive. It could well be that we have the following preference ordering between rules:

$$
\begin{aligned}
& R_{0}: g_{0} \leftarrow g_{1}, g_{2} \\
& R_{1}: g_{0} \leftarrow \neg g_{1} \\
& R_{2}: g_{0} \leftarrow \neg g_{2}
\end{aligned}
$$

with $R_{0} \succeq\left\{R_{1}, R_{2}\right\}$

In that case, it is clearly not possible to evaluate on a single point of view. It is only possible to say that we would prefer an action satisfying both $g_{1}$ and $g_{2}$, rather than only $\neg g_{1}$ or $g_{2}$. Only when the set of rules exhibit a very specific structure is it possible to interpret goals as proper criteria. In general however, this approach is more expressive and cater for preference models where "coalitions" of criteria are considered, which makes the comparison more difficult.

\subsection{Discussion}

In the previous section we have discussed several approaches to argument-based decision-making. What we have seen is that each approach is rather marginally different from the other ones, but that, by making explicit different steps of the process, they focus on different aspects of the process. Fox and Parsons are the only ones to explicitly represent the justification of a value assignment, however, they do not fully explore this avenue; and hardwire the possibility of having different criteria. Atkinson makes this latter distinction clear, but on the other hand, do not cater for an explicit representation of all the justifications of the value assignment (this only rely on the logical satisfaction: a goal is reached or not, which justifies the value assignment). In this case, it is not possible to represent or indeed challenge the preference structures used. Amgoud et al. also rely on the logical satisfaction of goals to justify the value assignment, but the goals are ordered in a way that indeed allows to refine the preference structure, to express various degrees of satisfaction of a goal. Still, this is directly encoded in the knowledge base and cannot be discussed in the process. Also, by using a bipolar scale, they constrain the syntax of goals and prevent themselves from using the full expressivity provided by the logic. Overall, it is important to emphasize that the definition of the argument scheme is of primary importance: by expliciting the inference steps of an argument, we also define what counts as valid "critical question", that is how arguments will interact with each others (how they can be attacked and so on). 
There are, on the other hand, many similarities between these approaches. First, the evaluation is made possible by an explicit representation of the consequences of the action. By relying on logic to represent these states of affairs, it is more expressive than the ordered scale that is usually used in decision models. One further possibility that is offered by this representation is that profile may be only partially defined, whereas in decision models you would require each action to be evaluated on each different criteria.

The third, perhaps most striking similarity being that they all rely on a method of intrinsic evaluation, and use more or less explicitly a profile. In decision models, on the other hand, the canonical case is the pairwise evaluation, that is, actions are evaluated against each others, and not against a profile. Although the use of profile can be justified, it has some consequences and drawbacks that, we feel, is important to emphasize:

- the adoption of a profile makes very important the definition of the neutral point, that is, the frontier (or more generally zone). In particular, a seemingly insignificant modification of the frontier can have tremendous consequences (as we shall see in the next section).

- in the context of multiparty decision-making, the problem is made even more thorny because it also generates potential conflicts as to what should count as positive or negative arguments, when agents would maybe more easily come up with an agreement if two alternatives were compared.

To elaborate on the point mentioned above, we refer to a recent discussion on the UAI (Uncertainty and Artificial Intelligence) list where the problem of "where to draw the line" emerged as a thread of discussion? A very illustrative real example of this problem was given, as reported here:

The contested 2000 US Presidential election and the question of "hanging chads." [...] in many instances the perforation was partial - leaving a hanging chad, a scrap of paper hanging from the voting card. So how was one to decide whether or not a partial perforation was or was not a vote for the position or person next to the perforation? One method, sometimes used, was to have the vote counter ask, "What intent does this perforation indicate?" Another approach was possible: It is useless or impossible to try to determine or guess the voter's intention. One must instead ask whether this perforation looks more like a vote than a non-vote ${ }^{3}$."

So for instance two agents could discuss whether a given value should count as a positive goal or a negative argument, one arguing that this is not high enough a mark to be counted as a positive argument... while, it would be more practical to simply ask the agents to simply say whether they prefer an alternative versus another wrt. this given criterion.

\footnotetext{
${ }^{3}$ [P. Tiller, post on UAI list in response to L. Zadeh]
} 


\section{Aggregation}

Once you have decided what counts as arguments pro and con, for each possible decision, it is necessary to aggregate them to eventually decide what alternative to select. At this point, there is an important question to be asked: how is it that you handle potential interactions between arguments that refer to different criteria?

- if you assume that these interactions do not exist, or do not take them into account, then you must first aggregate arguments independently on each criterion, and then aggregate the resulting criteria using a given operator;

- if you take into account these interactions, then it is necessary to design an aggregation process that will aggregate arguments labelled by criteria.

There are many rational ways to aggregate sets of pro and cons. Bonnefon and Fargier [BF06] offer a nice overview of different possible approaches. These approaches take into account the fact that the arguments are bipolar and qualitative. The importance of arguments is described on totally ordered scale of magnitude. In order to compare these qualitative, bipolar sets, Bonnefon and Fargier present several procedures: the Pareto comparison (sets of arguments are compared as as a problem of bi-criteria decision), the implication rule (this rule focuses on the most important arguments in the situation), the cardinality rules (based on a levelwise comparison by cardinality), and so on. The characterization of these rules was introduced in [DF05], and [BF06] present an extensive empirical assessment of the descriptive validity of these rules.

What Amgoud and al. show in [ABP05] is that it is possible to retrieve various classical aggregation operators in their framework. They propose to compare decision in terms of positive and negative arguments (using a complex scheme for evaluating the strength of argument, which depends on three parameters : the certainty level, the importance degree of the criterion, and the (dis)satisfaction degree of the criterion). Two principles based on preference relation between the arguments are proposed : promotion focus (take into account only the supporting arguments) and preventing focus (considers only the arguments against decisions). They show that the presented framework captures different multiple criteria decision rules to select the best decision. The rule for the choice is characterized by the fact that the criteria have or not the same importance level. In this approach however, the potential interaction between arguments, as analysed in the seminal work of Dung [Dun95], is not considered.

To the best of our knowledge, the value-based argumentation framework of $[\mathrm{BC} 02]$ is the only approach so far that proposes to compute the acceptable arguments from a set of labelled arguments. Indeed, argument refers to a given criterion (or "value" in the sense previously mentioned in the work of Atkinson [Atk05]). Argument systems, in the sense of Dung [Dun95], hence record interaction between arguments, possibly related to different values. Audiences are different ways to order those values. It is then possible to identify those arguments that will be accepted regardless of the chosen audience (objectively acceptable), while some others arguments can only be subjectively acceptable. 
In this case, the interaction between arguments pertaining to different criteria is fully recognized. The aggregation of values remains rather limited though, for it is only possible to order the values to reflect their degree of importance. So for instance it would not be possible to use an aggregation operator like the majority.

We conclude by a further remark related to the choice of the method of evaluation (intrinsic or pairwise). Both techniques may provide different, even contradictory, results, depending on the choice of the profile. Consider the example given below.

We assume that each criteria on an evaluation scale form 0 to 9 . We take the acceptability profile $p=[5,5,5]$, meaning that the neutral point on each criteria is 5 , and consider the following performance table :

\begin{tabular}{|c|c|c|c|}
\hline & $g_{1}$ & $g_{2}$ & $g_{3}$ \\
\hline$a$ & 8 & 6 & 8 \\
\hline$b$ & 7 & 4 & 2 \\
\hline$c$ & 9 & 7 & 4 \\
\hline
\end{tabular}

We will now use the following notation: $a \succeq b=[+,-,+]$, to specify that we have two arguments for the proposition that $a$ is preferred to $b$ (justified by the fact that the neutral point is reached on the first and third criteria), and one argument against it (justified by the fact that the neutral point is reached on the second criteria). We will now compare the results obtained by aggregation, comparing the cases where an intrinsic or pairwise evaluation is used.

- in the case of intrinsic evaluation, we get $a \succeq p=[+,+,+]$ and $c \succeq p=$ $[+,+,-]$. Then any rational aggregation method will give the outcome that $a \succeq c$.

- In the cas of a pairwise comparison (we don't need to use the profile then), we have on the other hand $a \succeq c=[-,-,+]$ (majority), hence $a \nsucceq c$

This simply illustrates that both methods may return contradictory results, which is easily explained by the fact that the categorisation as argument pro or con may make the preference model rather coarse-grained (of course, we do not discuss here the possibility of using a more detailed bipolar scale, as mentionned earlier in this paper).

\section{Conclusion}

There has recently been many proposals to adopt an argumentative approach to decision-making. As we feel that the underlying assumptions made in these different approaches is not always clearly stated, we reviewed these works, taking a more classical decision theory perspective, and even more precisely a multicriteria perspective. It appears that these approaches seem to have much to offer to decision models, because they allow more expressivity in the specification of 
agents' preferences, because they naturally cater for partial specification of preferences, and because they make explicit many aspect that are usually somewhat hidden in decision models. On the other hand, the typically intrinsic evaluation used in these approaches is not always the most appropriate, and it is not always clear how the multicriteria feature is taken into account when it comes to aggregating several arguments that may potentially interact and refer to different criteria.

\section{References}

[ABCM06] K. Atkinson, T.J.M. Bench-Capon, and S. Modgil. Argumentation for decision support. In DEXA, pages 822-831, 2006.

[ABP05] L. Amgoud, JF. Bonnefon, and H. Prade. An Argumentation-based Approach to Multiple Criteria Decision. In 8th European Conference on Symbolic and Quantitative Approaches to Reasoning with Uncertainty, ECSQARU'2005 , Barcelona, pages 269-280. LNCS, 06-08 juin 2005.

[aC96] C. Bana a Costa. Les problmatiques de l'aide la dcision : vers l'enrichissement de la trilogie choix-tri-rangement. Recherche Operationnelle, 30(2):191-216, 1996.

[AP06] L. Amgoud and H. Prade. Explaining qualitative decision under uncertainty by argumentation. In $A A A I, 2006$.

[Atk05] K. Atkinson. What Should We Do? : Computational Representation of Persuasive argument in practical reasoning. PhD thesis, Department of Computer Science, University of Liverpool, Liverpool, UK, 2005.

[Atk06] K. Atkinson. Value-based argumentation for democratic support. In P.E Dunne and T.J.M. Bnech-Capon, editors, Computational models of natural arguments, Proceedings of the International Conference on Computational Models of Natural Argument(COMMA 2006), volume 144, pages 47-58, Amesterdam, The Netherlands, 2006. IOS Press.

[BC02] T.J.M. Bench-Capon. Value-based argumentation frameworks. In NMR, pages 443-454, 2002.

[BF06] J.F Bonnefon and H. Fargier. Comparing sets of positive and negative arguments : Empirical assessment of seven qualitative rules. In G. Brewka, editor, Proceedings of 17th European Conference on Artificial Intelligence(ECAI2006), Riva di Gard, Italy, August 28- Septempber 1 2006. IOS Press.

[BG96] B. Bonet and H. Geffner. Arguing for Decisions: A Qualitative Model of Decision Making. In F. J. e. E. Horwitz, editor, 12th Conference on Uncertainty in Artificial Intelligence (UAI'96), pages 98-105, 1996.

$\left[\mathrm{BMP}^{+} 00\right]$ D. Bouyssou, T. Marchant, M. Pirlot, P. Perny, A. Tsoukiàs, and Ph. Vincke. Evaluation and decision models: a critical perspective. Kluwer Academic, Dordrecht, 2000.

[BS02] V. Belton and T. Stewart. Muliple Criteria Decision Analysis: An Integrated Approach. Kluwer Academic, Dordrecht, 2002.

[CLS05] Claudette Cayrol and Marie-Christine Lagasquie-Schiex. Graduality in argumentation. Journal of Artificial Intelligence Research, 23:245-297, 2005.

[DF05] D. Dubois and H. Fargier. On the qualitative comparison of sets of positive and negative affects. In ECSQARU, pages 305-316, 2005. 
[Dun95] P. M. Dung. On the Acceptability of Arguments and its Fundamental Role in Nonmonotonic Reasoning, Logic Programming and n-person games. Artificial Intelligence, 77(2):321-358, 1995.

[FP97] J. Fox and S. Parsons. On Using Arguments for Reasoning about Actions and Values. In John Doyle and Richmond H. Thomason, editors, Working Papers of the AAAI Spring Symposium on Qualitative Preferences in Deliberation and Practical Reasoning, pages 55-63, Menlo Park, California, 1997. American Association for Artificial Intelligence.

[FP98] J. Fox and S. Parsons. Arguing about beliefs and actions. In A. Hunter and S. Parsons, editors, Applications of Uncertainty Formalisms. Springer Verlag, Berlin, 1998.

[KP98] N.I. Karacapilidis and D. Papadias. Hermes: Supporting argumentative discourse in multi-agent decision making. In $A A A I / I A A I$, pages 827-832, 1998.

[MM07] M. Morge and P. Mancarella. The hedgehog and the fox. an argumentation-based decision support system. In Proc. of the Fourth International Workshop on Argumentation in Multi-Agent Systems, 2007.

[Mod06] S. Modgil. Value based argumentation in hierarchical argumentation. In 1st International Conference on Computational Models of Argument, Septembre 2006.

[PJ98] S. Parsons and N.R. Jennings. Argumentation and multi-agent decision making. In Proceedings of the AAAI Spring Symposium on Interactive and Mixed-Initiative Decision Making, pages 89-91, Stanford, USA, 1998.

[Pol87] J.L. Pollock. Defeasible reasoning. Cognitive Science, 11:481-518, 1987.

[PV02] H. Prakken and G. Vreeswijk. Logical systems for defeasible argumentation. In D. Gabbay and F. Guenthner, editors, Handbook of Philosophical Logic, second edition, volume 4, pages 219-318. Kluwer Academic Publishers, 2002.

[Tso] A. Tsoukiàs. On the concept of decision aiding process. Annals of $O p$ erations Research. To appear; appeared previously as DIMACS 2003-38 technical report, Rutgers University.

[Wal96] D.N. Walton. Argumentation schemes for Presumptive Reasoning. Mahwah, N. J.,Erlbaum, 1996. 